\title{
The Potential Role of Clinical Metagenomics in Infectious Diseases: Therapeutic Perspectives
}

\author{
Camille d'Humières ${ }^{1,2} \cdot$ Maud Salmona $^{3,4} \cdot$ Sarah Dellière ${ }^{5,6} \cdot$ Stefano Leo $^{7,8} \cdot$ Christophe Rodriguez $^{9,10}$. \\ Cécile Angebault ${ }^{9,11}$ - Alexandre Alanio ${ }^{5,6}$. Slim Fourati ${ }^{9,10}$. Vladimir Lazarevic ${ }^{7,8}$. Paul-Louis Woerther ${ }^{9,11}$. \\ Jacques Schrenzel ${ }^{7,8} \cdot$ Etienne Ruppé ${ }^{1,2}$ (B)
}

Accepted: 7 July 2021 / Published online: 30 July 2021

(C) The Author(s), under exclusive licence to Springer Nature Switzerland AG 2021

\begin{abstract}
Clinical metagenomics $(\mathrm{CMg})$ is the process of sequencing nucleic acid of clinical samples to obtain clinically relevant information such as the identification of microorganisms and their susceptibility to antimicrobials. Over the last decades, sequencing and bioinformatic solutions supporting $\mathrm{CMg}$ have much evolved and an increasing number of case reports and series covering various infectious diseases have been published. Metagenomics is a new approach to infectious disease diagnosis that is currently being developed and is certainly one of the most promising for the coming years. However, most $\mathrm{CMg}$ studies are retrospective, and few address the potential impact CMg could have on patient management, including initiation, adaptation, or cessation of antimicrobials. In this narrative review, we have discussed the potential role of $\mathrm{CMg}$ in bacteriology, virology, mycology, and parasitology. Several reports and case-series confirm that CMg is an innovative tool with which one can (i) identify more microorganisms than with conventional methods in a single test, (ii) obtain results within hours, and (iii) tailor the antimicrobial regimen of patients. However, the cost-efficiency of $\mathrm{CMg}$ and its real impact on patient management are still to be determined.
\end{abstract}

\section{Key Points}

Clinical metagenomics is an emerging diagnostic tool in infectious diseases.

Clinical metagenomics has the potential to identify unexpected microorganisms with no prior assumption (including fastidious ones) and to infer their susceptibility to antimicrobials.

Clinical metagenomics could have an impact on administered antimicrobial therapies.

Etienne Ruppé

etienne.ruppe@inserm.fr

1 Université de Paris, IAME, INSERM, 75018 Paris, France

2 AP-HP, Hôpital Bichat, Laboratoire de Bactériologie, Hôpital Bichat-Claude Bernard, 46 rue Henri Huchard, 75018 Paris, France

3 Unité de Paris, INSERM U976, Insight Team, 75010 Paris, France

4 AP-HP, Hôpital Saint-Louis, Laboratoire de Virologie, 75010 Paris, France

5 AP-HP, Hôpital Saint-Louis, Laboratoire de Parasitologie-Mycologie, 75010 Paris, France

\section{Introduction}

Clinical metagenomics ( $\mathrm{CMg}$ ) is the process of sequencing nucleic acids of clinical samples to obtain clinically relevant information such as identifying microorganisms and susceptibility to antimicrobials. $\mathrm{CMg}$ is a new field whose first application was published in the 2010s. Since then, the world has witnessed the development of more affordable and diverse next-generation sequencing machines. Their bioinformatics (solutions tools to convert DNA sequences into biological signals) have progressed in terms of DNA exploitation possibilities. Accordingly, clinical microbiologists are

6 Molecular Mycology Unit, Institut Pasteur, CNRS UMR2000, 75015 Paris, France

7 Faculty of Medicine, CMU, University of Geneva, Geneva, Switzerland

8 Service of Infectious Diseases, Genomic Research Laboratory, Geneva University Hospitals, Geneva, Switzerland

9 Département de Microbiologie, AP-HP, Hôpital Henri Mondor, 94000 Créteil, France

10 INSERM U955, Université Paris-Est, 94000 Créteil, France

11 Université Paris Est Créteil, Ecole Nationale Vétérinaire d'Alfort, USC ANSES, EA7380 Dynamic, 94000 Créteil, France 
all the more interested in metagenomic sequencing for clinical samples [1-6], mostly those facing a diagnostic dead-end despite extensive testing. One of the upsides of $\mathrm{CMg}$ over conventional methods is the capacity to detect microorganisms in an exhaustive manner without any presupposition. Over the past few years, several case reports and series have been published [7-10], many supporting the potential relevance of using $\mathrm{CMg}$ in a routine setting. However, few studies have shown the added value (including the adaptation of therapeutic regimen) of $\mathrm{CMg}$ as compared with conventional methods and the actual clinical impact of $\mathrm{CMg}$ remains to be assessed. In this narrative review, we aimed at (i) summarising the main wet lab and dry lab protocols of $\mathrm{CMg}$ and (ii) discussing the potential impact $\mathrm{CMg}$ could have on therapeutics (see Table 1).

\section{Wet Lab Experiments}

\subsection{Pre-extraction Step}

$\mathrm{CMg}$ can potentially be applied to any type of samples, provided that a sufficient amount of nucleic acids can be extracted [11]. The first step, called pre-extraction step, consists of accessing the nucleic acids of the potentially present microorganisms by breaking their cell membranes and walls. It typically includes (i) lysis of the microorganism (fungi, Gram-positive bacteria, mycobacteria, etc.) external structures and (ii) enrichment of the lysate with the nucleic acid of interest. Usually, a combination of different types of lysis methods are used, including chemical (detergent-based, e.g., sodium dodecyl sulfate), enzymatic (e.g., proteinase $\mathrm{K}$ or lysozyme), and mechanical lysis (beads beating, using beads of various size and composition [12]). Given that chemical lysis combined with proteinase $\mathrm{K}$ is an effective way to obtain DNA from Gram-negative bacteria and viruses, adding mechanical lysis greatly improves recovery yields in mycobacteria and Gram-positive bacteria, and is mandatory in fungi [13]. In any case, enrichment is typically used for the characterisation of microorganisms. Removing free DNAs and RNAs from the lysate using nucleases improves sequencing efficacy. However, this step greatly reduces the amount of nucleic acids of a particular microorganism (such as Pseudomonas) and should therefore be considered with caution $[14,15]$. Besides, samples can include a significant concentration of human cells (typically leukocytes in infection sites samples) and given that the human genome is larger than that of bacteria and yeasts by a $10^{3}$ magnitude, human DNA depletion can be considered [16, 17]. qPCR assays targeting bacterial 16S rRNA and human beta-actin genes may then be applied to determine the ratio of bacterial to host DNA [16].

\subsection{Nucleic Acids Extraction}

Nucleic acid extraction is typically performed using magnetic beads-based kits, which give good qualitative yields of nucleic acids for sequencing and can be automated [18]. For such, there are two options to consider: the extraction of RNA (followed by a retrotranscription into cDNA) to document the presence of RNA viruses or the host's gene expression profile, and the use of a kit that softly fragments the genomes when long reads are needed. Library preparation refers to the steps where raw nucleic acids are converted to a material ready to be sequenced. Two main approaches can serve the purpose: amplicon and shotgun. With the amplicon approach, particular care should be taken when choosing the primers to use. Traditional targets are the variable regions v1-v4 loops of the 16S rRNA encoding gene for bacteria, and internal transcribed spacers (ITS) 1 and 2 for fungi [19, 20]. With the shotgun approach, no prior amplification is needed. Nucleic acids are then prepared so that they might be sheared according to the size of the expected reads, and attached to adapters and sometimes to primers.

\subsection{Nucleic Acid Sequencing}

Sequencing offers two main alternatives: a high number of short reads (Illumina, ThermoFisher) or a small number of long reads (Oxford Nanopore Technologies, Pacific Biosciences). In amplicon-based metagenomics, short-read sequencing with a low output (e.g., $<50,000$ reads, $2 \times 250$ bp length) is usually chosen [21]. As for amplicon long-read sequencing ( $>1000 \mathrm{bp}$ ), it provides better accuracy of species characterisation since all variable regions are covered [22]. In a shotgun approach, high-throughput short-read sequencing ( $>10$ million reads) is most commonly used because the documentation often requires a very high sequencing depth to provide sufficient sensitivity, especially if the sample has not been depleted of human DNA [23]. Conversely, low-throughput, long-read sequencing can provide a higher specificity of species detection. When genome reconstruction is desired, long-read sequencing, possibly coupled with short-read sequencing, appears to be a promising option [24].

\subsection{Controls}

Irrespective of the chosen approach, using appropriate controls is mandatory. There must be at least a no template control (NTC) prepared in the same conditions of sample collection, and a positive control, preferably quantitative and containing the widest possible microbial diversity [25]. It is also possible to include a spiked internal control (DNA and RNA) to all samples to ensure that each step is correctly carried out [26]. 


\section{Principles of Bioinformatics Applied to Clinical Metagenomics (CMg)}

The adjustment of $\mathrm{CMg}$ to meet clinical microbiology requirements calls for the development of bioinformatics pipelines to achieve the best performance in terms of sensitivity and specificity. To date, most $\mathrm{CMg}$ pipelines are custom-made and their functionality, in comparison with each other, has been poorly assessed despite recent initiatives like CAMI project [27] and ring trials [28]. As the current development of $\mathrm{CMg}$ is based mainly on shotgun sequencing with pan-pathogen detection approach, we will focus in this section on bioinformatics pipelines using shotgun metagenomics data as input. Of note, such data require (i) substantial calculation and storage resources with respect to the volume of sequences generated by next-generation sequencing (NGS) machines, (ii) bioinformatics skills, even though user-friendly interfaces such as Galaxy [29] or NanoGalaxy [30] have been made available.

\subsection{Taxonomic Assignment}

A typical $\mathrm{CMg}$ bioinformatics pipeline (Fig. 1) starts by cleaning the sequences to keep the desired quality and size, followed by removing duplicate sequences, subtracting reads aligned to the human genome (except if the host's transcriptome is considered), and then aligning the remaining sequences to reference microbial databases in order to perform taxonomic assignment. This last step is critical since the specificity and sensitivity of the assignment pipeline will crucially rely on the exhaustiveness and curation of databases as well as on the stringency of the software in matching sequences to reference databases (e.g., alignment or $\mathrm{k}$-mer based, see below). Taxonomy classification errors in databases may lead to false assignations and perhaps adverse outcomes for the patient. Likewise, a database that does not include the infection-causative pathogen would give a false negative result, with detrimental consequences as well. As there is no such perfect combination between a database and a software, a multiple approach using diverse databases and software types is warranted. For instance, MetaPhlAn2 [31] is a specific taxonomic tool that relies on conserved sequences within species onto which reads are aligned. However, it does not cover all possibly existing microorganisms and may miss subdominant, under-sequenced species [27]. A more sensitive approach is to use alignment tools (e.g., BLAST [32], Bowtie2 [33] or BWA-MEM [34]) and broader, non-specific databases such as NCBI RefSeq or nt, but at a more expensive computational cost. In complement, more sensitive tools can be used such as k-mer-based tools (e.g., Kraken [35], Clark [36] or Centrifuge [37]). With $\mathrm{k}$-mer approach, fast classification is performed using exact matching of short words of length $\mathrm{k}$ (referred to as k-mers). Reads can also be assembled with software like metaSPAdes [38] for short reads or metaFlye [39] for long reads in order to reconstruct genomes and identify genes.

\subsection{Secondary Analyses}

Once microorganisms are identified, other traits can be sought. In microbiology, susceptibility to antimicrobials is as equally important as the taxonomic assignment, if not more, since the antimicrobial choice depends on it. Detecting antimicrobial resistance determinants and inferring a phenotype is a task $\mathrm{CMg}$ can potentially address. Nonetheless, knowing the genetic determinants is required in order to infer the proper genotype from the phenotype. For bacteria, there are several databases for antibiotic resistance genes (ARG) [40-42] and for resistance-associated mutations, and genotype-to-phenotype inference works well for some specific combinations of pathogens-antibiotics when single genomes are tested [42, 43]. Genotypic antibiotic susceptibility testing should become a routine tool for Mycobacterium tuberculosis provided that NGS devices are available [44]. However, dealing with metagenomic data is challenging since more than one bacterial genome can be present. Since many ARG are embedded in mobile genetic elements, connecting each ARG to its host is a challenge bioinformatics has not yet overcome. Besides ARG, other genes of interest such as virulence genes, or those related to phylogenetic analysis can be analysed provided that sufficient sequencing depth is achieved in order to span the whole microorganism genome.

RNA sequencing helps detect potential RNA viruses and gives access to mRNA sequences of the host, i.e., allows analysis of the human transcriptome whilst searching for pathogens. Recently, Langelier et al have shown that the analysis of the human transcriptome could be leveraged to identify pulmonary infection, in comparison with noninfected samples, thereby offering clinicians a decision tool to uphold antimicrobial drugs or stop them [3]. In Lyme disease, specific gene expression signature suggests that a panel of selected human host-based biomarkers could help in the diagnosis [45]. However, integrating host RNA analysis in a bioinformatics pipeline requires additional resources and the implementation of machine-learning solutions [46]. Another interesting approach is performing metagenomic analysis of microbiota in non-sterile samples (e.g., respiratory tract sample). It has been shown that oropharyngeal flora associated with lung infection have a lower diversity index than those of non-infected patients. This original approach can also improve the capacity to distinguish infections from colonisation [3]. Similarly, the quantification of microorganisms using thresholds expressed in colony-forming units per $\mathrm{mL}$ can be of help in bacteriology (e.g., in respiratory or 

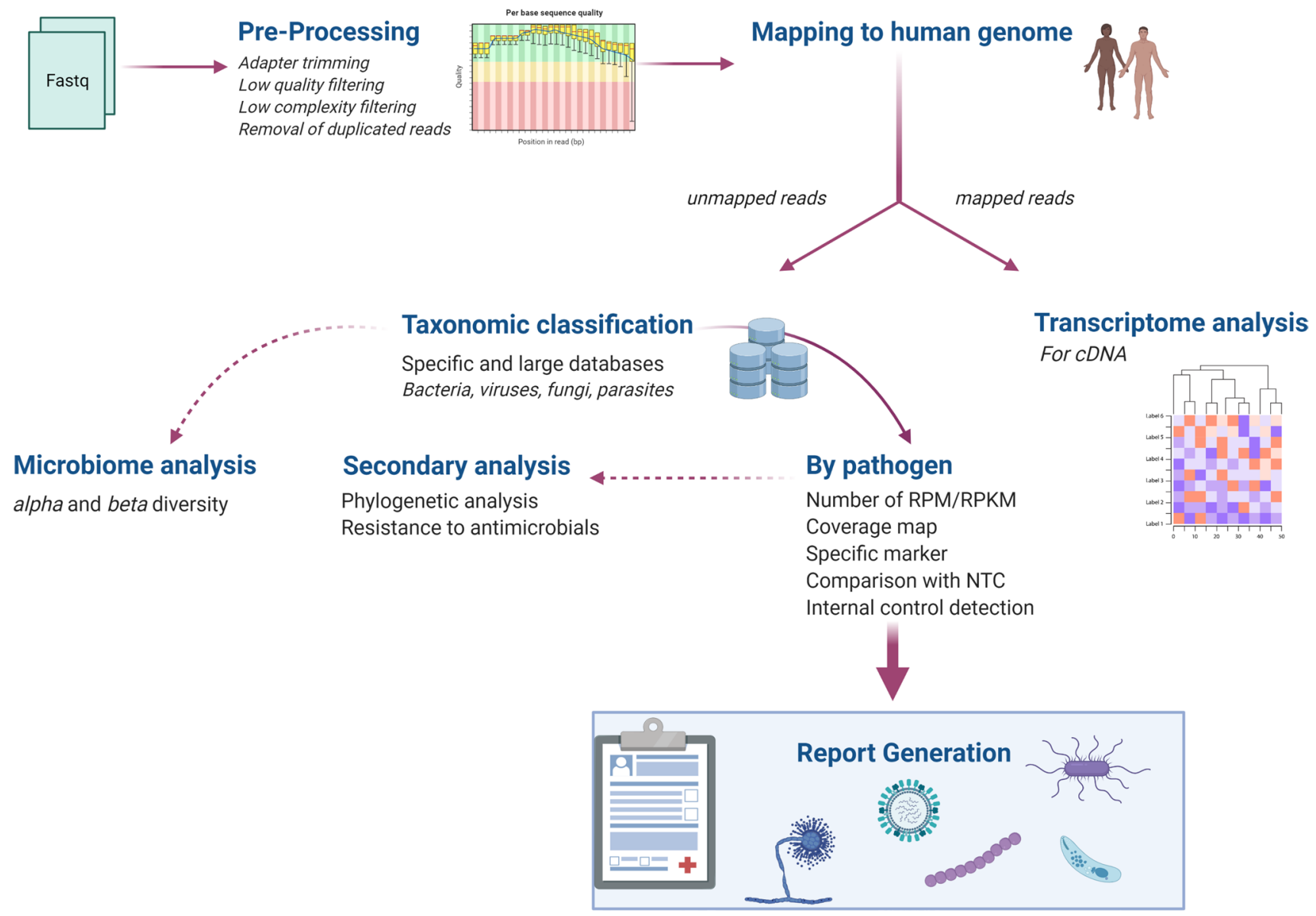

Fig. 1 Schematic representation of an example of a clinical metagenomics bioinformatics pipeline from reads processing to clinical reporting. $N T C$ no template control, RPKM reads per kilobase million, RPM reads per million (created with BioRender.com)

urinary samples). Metagenomic sequencing provides relative quantification (expressed as a ratio). Albeit, transforming relative concentrations into absolute microorganisms concentrations, as proposed by Blauwkamp et al [4] using molecules of microbe-specific cfDNA per microlitre of plasma (MPM), provides perspectives to distinguish actual pathogens from commensals or contaminants.

\subsection{Contamination Challenge and Pipeline Validation}

To avoid false positive results, detection criteria aimed at distinguishing microorganisms actually present in the sample from contaminants play a key role. To that end, it is possible to consider the number of reads per million (RPM) or RPKM (reads per kilobase million) assigned to a microorganism with respect to the number of reads of the same microorganism found in the negative control (NTC). For instance, SURPI + pipeline [47] reports pathogens have an RPMsample / RPMNTC $>10$. Other strategies rely on the probability of finding a microorganism in a sample and comparing results with a set of negative controls [48, 49], the genome coverage of the microorganism or the identification of microorganism-specific genomic markers.

\subsection{Report}

It is important to produce a report that is understandable, meaningful, and actionable by both microbiologists and clinicians. Ideally, some bioinformatics output markers should be provided such as the estimation of genome coverage, the relative abundance, and the degree of identification confidence. In a certification perspective, the software and related versions should also be provided. If antibiotic resistance determinants are needed (in cases where genotype-phenotype inference is simple), then a short description (e.g., to which antibiotic they have been reported to confer resistance) should also be given [40, 42]. 


\section{Application of CMg in Bacteriology}

\subsection{CMg May Play a Therapeutic Role by Detecting Unexpected or Fastidious Pathogens}

Central nervous system (CNS) infections are amongst the leading infections explored by $\mathrm{CMg}$. Overall, etiological diagnosis of encephalitis is not reached in $\sim 50 \%$ of patients [50]. It is estimated that the prevalence of autoimmune encephalitis might equal that of infectious encephalitis [51], hence the need for new diagnostic tools to detect/rule out infections caused by rare or emerging microbes, and to adapt/stop anti-infectious agents (or other treatments) accordingly. The seminal study of neuroleptospirosis diagnosed by $\mathrm{CMg}$ [1] paved the way for the insertion of $\mathrm{CMg}$ in the medical investigations of CNS infections. Further data demonstrated that $\mathrm{CMg}$ had a sensitivity of $73-92 \%$ and a specificity of 96-99\%, depending on the pathogen detected in cerebrospinal fluid (CSF) [1]. More recently, Wilson et al included 204 critically-ill, idiopathic meningitis patients, with or without encephalitis [26], in whom 58 infections were diagnosed in 57 patients (27.9\%). Of these 58 infections, 13 (22\%) were diagnosed by $\mathrm{CMg}, 26$ (45\%) by conventional methods (11 by serology, 7 from tissue samples other than CSF, and 8 by direct testing), and 19 (33\%) by both approaches. Among the 13 infections diagnosed by $\mathrm{CMg}$ alone, healthcare was subsequently adjusted in nine of them including the antimicrobial regimen.

Bone and joint infections (BJIs) are complex infections that are often explored by $\mathrm{CMg}$. Thoendel et al sequenced 408 sonicate fluid samples drawn from resected hips and knee arthroplasties, of which 213 samples came from patients with infections and 195 from subjects without infection [52]. Compared with the results of sonicate fluid culture, $\mathrm{CMg}$ confirmed pathogens presence in $94.8 \%$ (109/115) of cases and revealed additional potential pathogens in $9.6 \%(11 / 115)$. In culture negative samples, pathogens were detected in $43.9 \%$ (43/98) of cases. Bacteria were rarely detected in samples from presumably aseptic failure cases (7/195 [3.6\%]). Comparable performances were found by other teams on samples drawn from prosthetic joints and other orthopaedic device infections or total knee arthroplasties $[53,54]$. CMg was also found suitable to detect (i) less common pathogens like M. tuberculosis or Brucella melitensis in a study conducted on patients with osteoarticular infections [55], or (ii) novel pathogens like Mycoplasma salivarium detected in a BJI [56].

\subsection{CMg May Play a Therapeutic Role by Reducing the Time to Actionable Results}

The time gap between the onset of an infection (e.g., bacterial) and the start of effective antibiotic therapy has often been correlated with the outcome, especially in intensive care [57]. Culture-based methods typically yield results within $24-72 \mathrm{~h}$ after sampling, whereas sequencing using Nanopore technologies (Oxford Nanopore Technologies, Oxford, UK) can provide results with a turnaround time of $<10 \mathrm{~h}[17,58,59]$. First cases of respiratory infection diagnosed by $\mathrm{CMg}$ faster than by culture were two pneumonia cases incurred by Pseudomonas aeruginosa and Staphylococcus aureus [59]. More recently, Charalampous et al used Nanopore sequencing on respiratory samples in the context of hospital-acquired infections [17]. After human DNA depletion, they detected respiratory pathogens with $96.6 \%$ sensitivity as compared with culture methods, with optimal turnaround time of $6 \mathrm{~h}$.

\subsection{CMg May Help Identify the Microorganism Responsible for Sepsis}

Faster time-to-results could also help in sepsis management. However, the low number of bacterial cells present in the blood sample in bloodstream infection (BSI) represents a significant challenge for CMg. Gyarmati et al applied metagenomic sequencing to 27 blood samples from nine patients with acute leukaemia and suspected BSI at different time points with respect to their exposure to antibiotics. The authors evidenced the presence of bacteria during fever but not after initiation of the antibiotic therapy [60]. However, common contaminants (mainly Cutibacterium acnes, Corynebacterium spp., and Staphylococcus spp.) were detected, which doubled the challenge to distinguish true pathogens from contaminants. In another study [48], 101 blood samples from immunosuppressed patients were sequenced and $\mathrm{CMg}$ displayed a significantly higher proportion of bacteria in those patients (mostly Pseudomonas spp., $n=17)$ as compared with conventional methods $(36 / 101$ [36\%] vs $11 / 101$ [11\%], respectively, $p<0.001)$. CMg has also been tested in 35 children with confirmed $(n=12)$ or suspected BSI (sepsis with negative blood culture, $n=23$ ) [61]. Among patients with confirmed BSI, bacteria were identified (at the family level) in eight patients $(8 / 12,67 \%)$. In patients with suspected BSI, 3 of the 23 samples yielded signals of microorganisms putatively causing the sepsis. CMg based on cell-free DNA sequencing such as that developed by Karius company (Redwood, CA) showed 93.7\% agreement with blood culture in a cohort of 350 patients with suspected BSI [4]. Moreover, it identified an independently adjudicated cause of the sepsis alert more often than all of the microbiological testing combined (169 aetiological 
determinations vs 132). However, the method significantly detected microorganisms in 38/167 (22.8\%) asymptomatic volunteers, stressing the need for further studies on cell-free DNA sequencing.

\subsection{CMg May Play a Therapeutic Role by Rapidly Adapting Antimicrobial Therapies}

Searching for resistance markers in $\mathrm{CMg}$ data requires a high sequencing depth, especially when host's DNA depletion is not applied [59]. In a study on a large number of respiratory samples using saponin-based host DNA depletion, Charalampous et al searched for antibiotic resistance genes (ARG) in metagenomic data of respiratory samples [17]. Of the 187 detected ARG (41 samples), 24 matched the phenotypic resistance observed in culture, and 14 were classified as relevant, but the drug was not tested by a clinical laboratory. Many of them (98/187) were suspected as originating from commensal bacteria (commonly recovered in proximal respiratory samples, e.g., sputa and endotracheal aspirations) whose DNA was found along with that of pathogens, hence the difficulty to connect ARG to their host in metagenomic data. In addition, some ARG were presumably not detected because of the insufficient genome coverage of the pathogens. Indeed, another study found a correct inference of antibiotic susceptibility that was achieved in $94.1 \%$ of the 24 BJI samples, where only one bacterium was found (and its genome sufficiently covered to detect ARG), and in $76.5 \%$ of samples where more than one bacterium was found in culture [62]. Using ARG genotype-to-phenotype inference to guide patient therapy is already challenging upon working on genomic data, especially when resistance arises from a change in gene expression (e.g., cephalosporinase-encoding gene AmpC). Dealing with metagenomic data brings complexity as linking an ARG to its host is not achievable unless cross-ligation is performed during the DNA extraction step. As for ARG shared by pathogens and commensals such as $m e c A$ in staphylococci, achieving a correct phenotypic inference remains difficult.

\section{Application of CMg in Virology}

\subsection{CMg Can Detect New or Unexpected Viruses}

Currently, the most commonly used techniques to diagnose viral infections target one or a limited number of viruses. The functionality of these traditional methods, such as PCR (including multiplex PCR) or serology, cannot be extended to discover new emerging viruses, unexpected viruses attributable to a given pathology, or viruses that are uncommon to a particular geographic area. Unlike bacteria whose $16 \mathrm{~S}$ rRNA gene can be targeted, there is no common gene to all viruses, and only shotgun sequencing offers unbiased detection of all viral genomes. Theoretically, with $\mathrm{CMg}$ it is possible to find unknown or unexpected viruses in clinical samples. In practice, numerous clinical cases or series have shown the success of this technique in diagnosing viruses in different types of infections including encephalitis, pneumonia, fever/sepsis, or even eye infections [46, 63-66].

\subsection{CMg Can Help Tailor the Antiviral Therapy}

Antiviral therapeutic panel is still very limited (except for HIV and viral hepatitis), therefore, diagnosing a viral infection by $\mathrm{CMg}$ often does not help implement a specific treatment. However, a few case reports have shown that diagnosing viruses via $\mathrm{CMg}$ made it possible to initiate a specific treatment. For instance, Murkey et al reported hepatitis E virus-associated meningoencephalitis diagnosed by $\mathrm{CMg}$ in a lung transplant recipient, which was successfully treated by ribavirin [67]. CMg also helped diagnose unexpected herpes zoster laryngitis, which incited clinicians to start acyclovir treatment and stop fingolimod [68]. A case of astrovirus meningoencephalitis was also reported, and the patient was successfully treated with ribavirin and interferon [69]. Another case of astrovirus encephalitis was similarly treated with ribavirin without success [70]. Recently a case of chronic Jamestown Canyon virus encephalitis diagnosed by $\mathrm{CMg}$ in a patient receiving rituximab has been reported [71]. In the absence of approved treatment, the patient was treated with intravenous immunoglobulin and favipiravir, albeit with an unfavourable outcome. Despite the abovementioned therapeutic failures, the diagnosis of these viral infections remains crucial in order to (i) stop unnecessary antiviral therapy and (ii) introduce approved or experimental therapeutic regimens as early as possible.

Another indication of $\mathrm{CMg}$ is the detection of dominant (high level) as well as minor (low level) markers of resistance to antiviral drugs. Provided the viral load is high enough, $\mathrm{CMg}$ potentially gives access to the full-length genome of the virus, and thus allows identification of resistance-associated substitutions (RASs) in patients infected with HIV (by analysing reverse transcriptase, protease, and integrase-encoding genes) or viral hepatitis (by analysing NS3, NS5A, and NS5B sequences) when antiviral treatment failure is suspected. Such indication has also been adapted for cytomegalovirus (by analysing UL97, UL54, and UL56 in letermovir failure), herpes simplex viruses, or influenza [72-74]. Identifying resistance-associated mutations in the targeted genes is of great interest to adjust treatment accordingly. 


\subsection{CMg Can Divert Treatment Towards Non-antimicrobial Drugs}

Diagnosing an aetiological agent by $\mathrm{CMg}$ in a case of infection can spare patients unnecessary investigations, invasive procedures, and empirical antibiotic therapy [75]. CMg can also guide the clinician to use immunomodulating therapies. In another word, the absence of infection as evidenced by $\mathrm{CMg}$, in addition to conventional tests, gives the clinician a supplementary argument to initiate an immunosuppressive therapy to treat a possible autoimmune disease [26, 76]. However, it is important to note that $\mathrm{CMg}$ might be less sensitive than targeted techniques for the detection of a particular pathogen $[1,4,26,52]$ stressing that $\mathrm{CMg}$ should not replace conventional methods.

\subsection{CMg Can Help Describe Genetic Diversity}

Another interesting use of $\mathrm{CMg}$ is the possibility of characterising the viral genetic diversity. Viruses, particularly RNA viruses, and to a lesser extent DNA viruses, rapidly adapt to their environment (immune pressure, antiviral treatment). In SARS-CoV-2 pandemic, $\mathrm{CMg}$ has been so useful in profoundly characterising the full-length genome of circulating variants, which allows researchers to analyse the selection of specific mutations towards immune response [77]. It has been shown to be relevant in characterising divergent genotypes within a virus genus, as Sanger sequencing might miss them [73, 78]. The potential of CMg to sequence the fulllength genome also provides a great opportunity to detect fitness-associated substitutions in $\mathrm{HCV}$-infected patients failing treatment [79].

\subsection{CMg Can Help Study Outbreaks}

Taxonomic assignment and phylogenetic analyses can also be carried out for epidemiological studies. $\mathrm{CMg}$ has been used successfully to characterise the epidemiology of Zika and Ebola viruses in several regions of the world [80, 81], which is pivotal for the surveillance of emerging viruses and the rapid implementation of wide-scale public health measures. The current SARS-CoV-2 pandemic, discovered and characterised by CMg [82], demonstrates the need for non-targeted screening of potential pathogens and their rapid characterisation.

\section{Application of CMg in Mycology and Parasitology}

\subsection{CMg May Help to Identify Fungi}

Application of CMg in medical mycology has not exceeded the research stage despite the long field of experience in ecology [83]. Addressing technical issues and methodological biases beforehand would enable researchers to associate fungal species to medical conditions [84]. However, this path is still not useful in clinical practice. Identifying fungi by shotgun metagenomics in sterile samples of cerebrospinal fluids or biopsies may provide a diagnosis and then significantly change the therapeutic strategy. Fungi are common contaminants of air and reagents; therefore, interpretation of results requires caution and multidisciplinary discussions. In respiratory invasive fungal diseases, especially aspergillosis and Pneumocystis jirovecii pneumonia, distinguishing colonisation/carriage from infection is crucial for proper patient management; however, currently there is no common consensus $[85,86]$. Some studies suggest that metagenomic analysis of fungal abundances with respect to the remaining microbiota together with a specific cut-off could be exploited for clinical diagnosis [87-89]. In a retrospective analysis performed by Hogan et al on 82 Karius tests, $\mathrm{CMg}$-positive results represented $61 \%$, albeit with poor clinical impact on cases (7.3\%). Three of the latter six cases were fungal diseases (aspergillosis, $n=1$; candidiasis, $n=1$ and mucormycosis, $n=1$ ) hence, the initiation of antifungal treatments [90].

\subsection{CMg May Be an Asset to Diagnose Rare and Fatal Parasitic Diseases}

Regarding parasitic infections, very few publications used CMg to diagnose parasitic cases. In 2015, Wilson et al reported a case of meningoencephalitis secondary to Balamuthia mandrillaris diagnosed post-mortally by $\mathrm{CMg}$ [91]. Similarly, a case of CNS angiostrongyliasis was diagnosed by $\mathrm{CMg}$ that led to the introduction of an appropriate treatment [9]. Unfortunately, the delayed treatment did not prevent death. Of note, the earlier the diagnosis of angiostrongyliasis, the better the prognosis. A rapid and reliable $\mathrm{CMg}$ diagnosis might be of great help in such life-threatening infections. Other publications reported parasitic diseases such as visceral leishmaniasis [10] and CNS angiostrongyliasis [26] for which the diagnosis was confirmed by $\mathrm{CMg}$ after first being suspected by conventional methods. Schneeberger et al also showed that shotgun CMg could help detect pathogens, including parasites, in faeces [92]. For instance, in two patients with persistent diarrhoea, $\mathrm{CMg}$ identified Ascaris lumbricoides and Schistosoma mansoni (patient 1), 


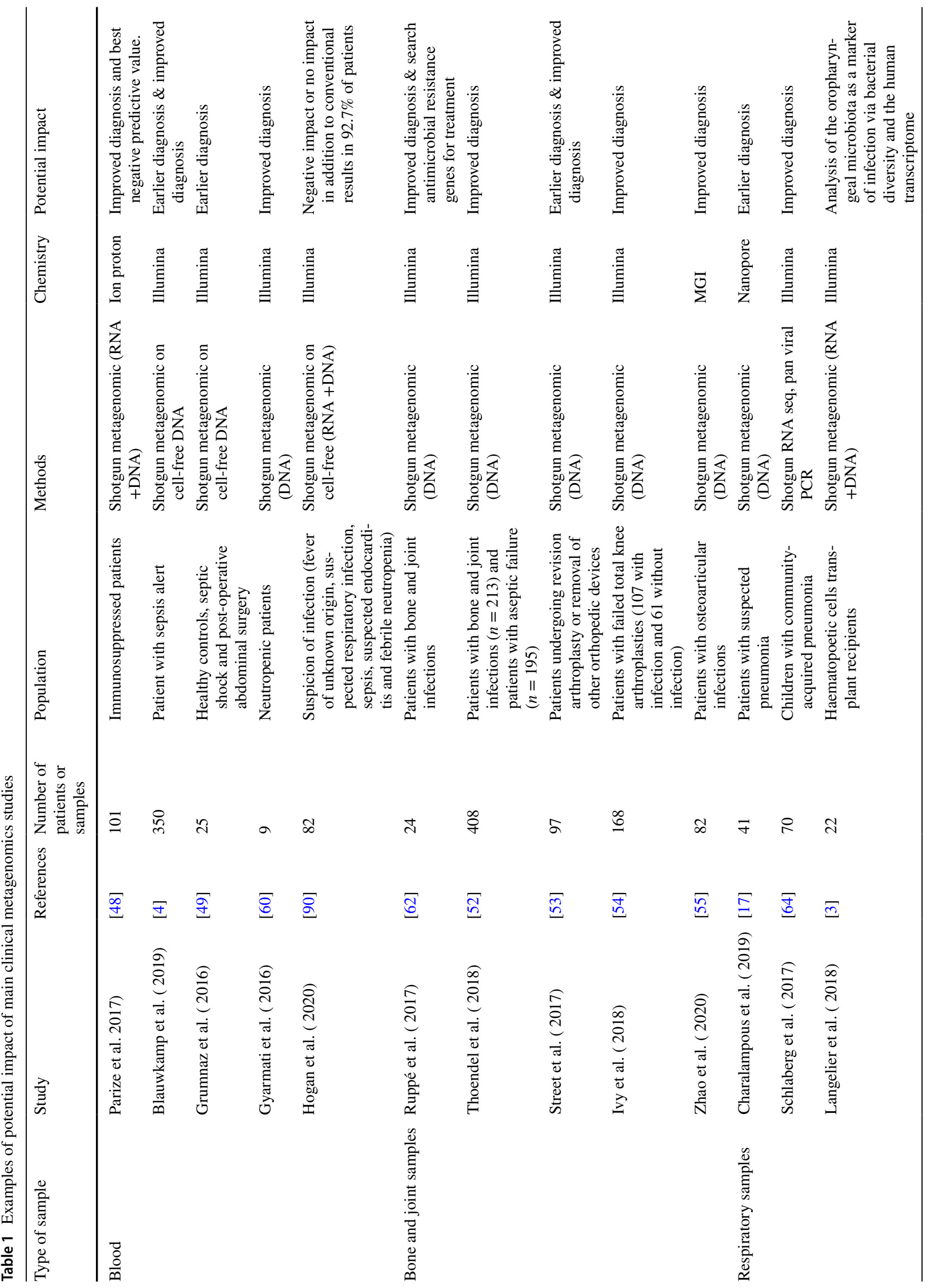




\begin{tabular}{|c|c|c|c|c|c|c|c|}
\hline 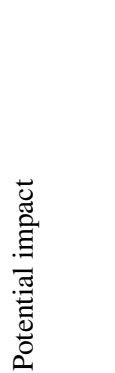 & 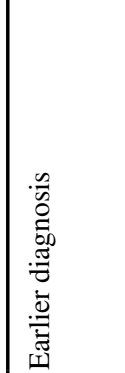 & 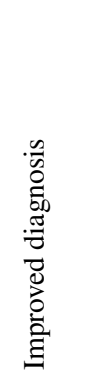 & 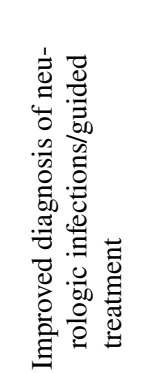 & 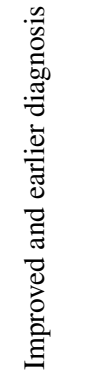 & 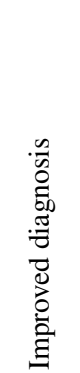 & $\begin{array}{l}\frac{x}{0} \\
\vec{n} \\
0 \\
\frac{0}{2}\end{array}$ & 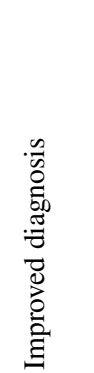 \\
\hline $\begin{array}{l}\grave{E} \\
\text { है } \\
\text { ह } \\
\text { U }\end{array}$ & 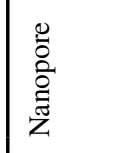 & 嗬 & 曽 & 祃 壳 & 萛 & 菲 & 蒷 \\
\hline 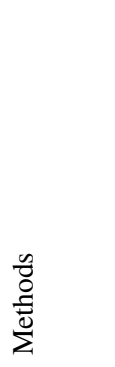 & 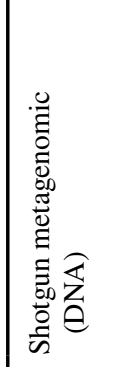 & 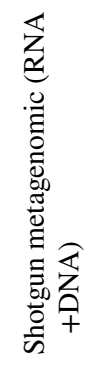 & 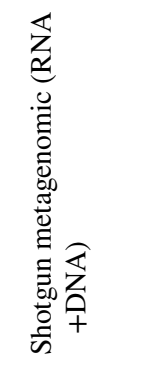 & 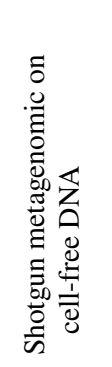 & 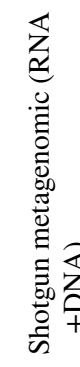 & 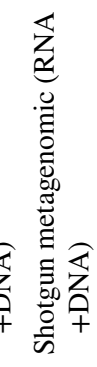 & 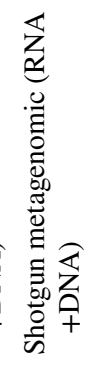 \\
\hline 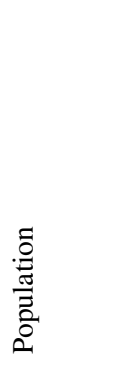 & 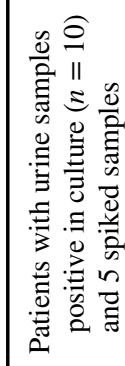 & 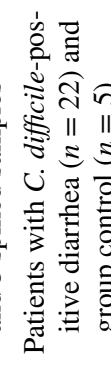 & 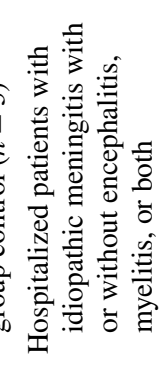 & 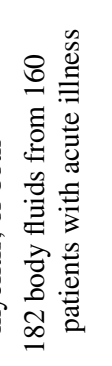 & 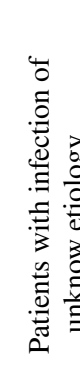 & 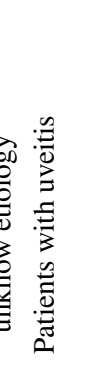 & 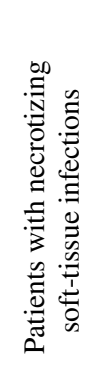 \\
\hline 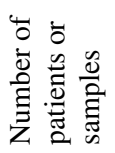 & $\simeq$ & $\hat{\lambda}$ & త్ & $\stackrel{8}{0}$ & $\cong$ & in & 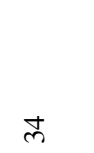 \\
\hline 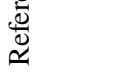 & 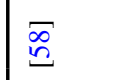 & $\sqrt{n}$ & $\stackrel{\Xi}{d}$ & $\underline{\sigma}$ & $\stackrel{\Xi}{\Xi}$ & 룽 & $\overline{\widehat{\theta}}$ \\
\hline 害 & 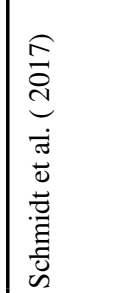 & 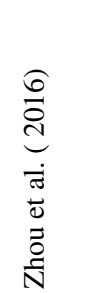 & 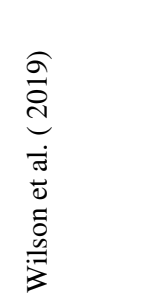 & 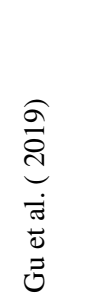 & 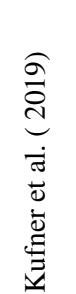 & 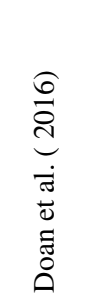 & 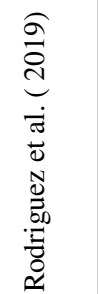 \\
\hline 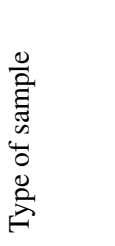 & $\stackrel{g}{\Xi}$ & $\begin{array}{l}\mathscr{U} \\
\tilde{U}\end{array}$ & 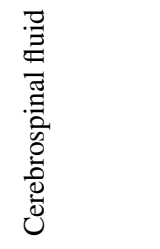 & 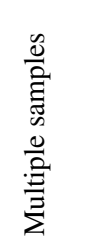 & & 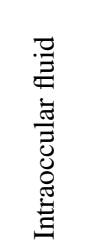 & 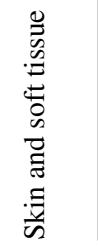 \\
\hline
\end{tabular}




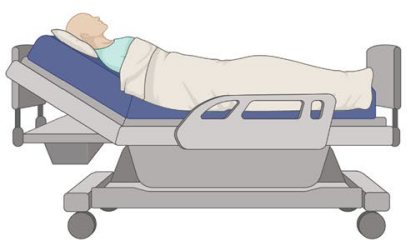

Clinical Metagenomics

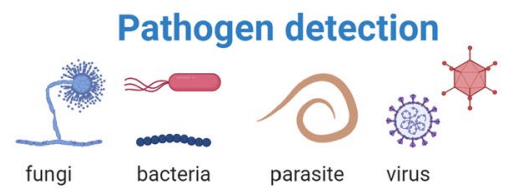

Antimicrobial resistance prediction

Virulence factor

Epidemiological studies

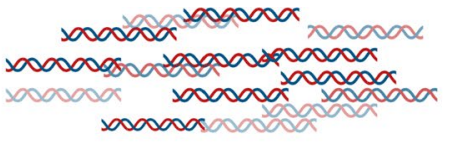

(1)

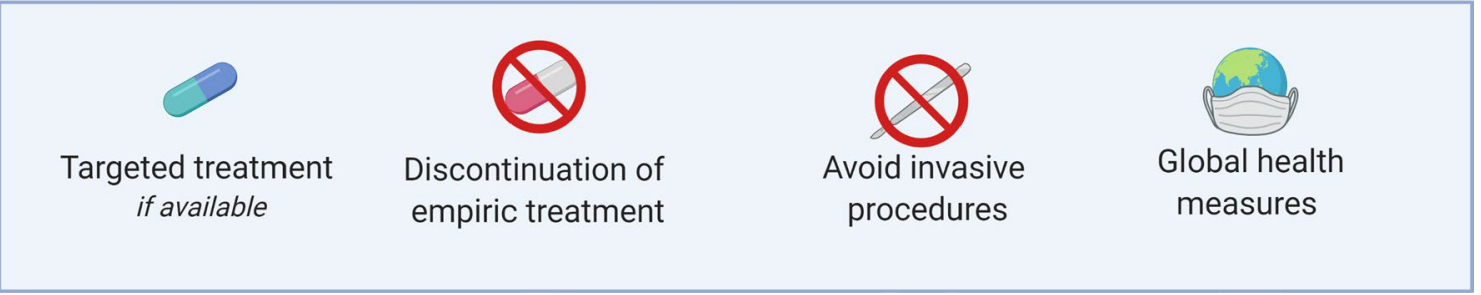

Fig. 2 Summary of the potential impact of clinical metagenomics (created with BioRender.com)

and Giardia intestinalis (patient 2). However, CMg missed G. intestinalis and Chilomastix mesnili in patient 1 . These reports illustrate the sensitivity and temporality issues of $\mathrm{CMg}$. In the 82 Karius tests reported by Hogan et al, no parasites were detected in blood [90].

\subsection{CMg May Have an Impact on the Antifungal Therapy}

Antifungal resistance in fungi is an emerging threat, especially in Candida spp. [93] and Aspergillus fumigatus [94], but few studies have investigated this issue using metagenomics, and none of them had a direct clinical impact [95-97]. Metagenomics could rapidly detect local prevalence of cyp51A resistance genotype in A. fumigatus, which is pivotal in determining first-line treatments [98]. Wholegenome sequencing (WGS) of Aspergillus isolates, which exhibit an unexplained azole-resistant phenotype, makes it possible to identify new mutations potentially responsible for resistance [96, 99]. Similarly, WGS of Candida isolates showing azole and echinocandin resistance can quickly identify mutations and putative resistance mechanisms [97, 100]. Regarding resistance detection in parasites, no publication on $\mathrm{CMg}$ has been made available to date. A few genomic studies focused on Plasmodium resistance detection, yet had no direct therapeutic implications [101]. WGS was also used by Doyle et al to describe a new locus associated with ivermectin resistance in a veterinarian nematode, Haemonchus contortus, which could help researchers improve their understanding of ivermectin resistance in human nematodes [102].

\subsection{CMg, a Field to be Developed for Mycological and Parasitological Purposes}

Overall, although $\mathrm{CMg}$ theoretically generates the same results as routine methods $[63,87]$, the complexity of the procedure and the need for specific protocols are currently preventing its routine implementation for medical mycology or parasitology. Moreover, parasites and fungi are usually present in low loads in infected specimens, which hinders their detection with less sensible methods. As eukaryotes, they share large parts of their genome with humans, thus some of fungal or parasitic DNA is filtered during bioinformatics trimming process incurring reduction of test sensitivity [86, 103]. Specific adaptation of $\mathrm{CMg}$ might thus be necessary for parasitic or fungal diagnostics. Finally, the high cost and technicity of $\mathrm{CMg}$ are as of today barely compatible with large-scale implementation in low-income areas where parasitic diseases often prevail.

\section{Conclusion and Perspectives}

As an emerging discipline crossing microbiology, infectious diseases, and bioinformatics, $\mathrm{CMg}$ has witnessed fast development over the last decade. The multiple sequencing tools together with the diverse bioinformatic solutions have provided $\mathrm{CMg}$ enthusiasts a vast array of possibilities to play with. Several case-series have been published, including some showing a potential impact on patient management. The place of $\mathrm{CMg}$ in the diagnostics arsenal has yet to be defined in order to use it to its best advantage. As $\mathrm{CMg}$ will gradually impose itself as a diagnostic tool of infections, 
standardisation of its methods (wet and dry lab steps) as well as randomised controlled trials are now needed; the aim is to elaborate strong recommendations on methodological key points, spanning of nucleic acid, and bioinformatics analysis. As for other tests, the position of $\mathrm{CMg}$ will most likely depend on the clinical context. Being highly versatile given the diversity of sequencing and bioinformatic solutions, $\mathrm{CMg}$ will enable researchers to address many challenges such as (i) identification of hard-to-culture or dead (due to a previous antimicrobial exposure) microorganisms (ii) fast diagnosis (iii) identification with no prior assumption of unexpected or new microorganisms (Fig. 2). $\mathrm{CMg}$ should also be positioned according to its cost-efficiency. CMg is more expensive than traditional culture or targeted PCR techniques. However, that could be balanced against (ii) savings in other tests and investigations (ii) using targeted therapies much earlier, and (iii) earlier patient discharge. For such, this may plead for a higher position of $\mathrm{CMg}$ in the diagnostic algorithm.

In conclusion, $\mathrm{CMg}$ is emerging as a promising diagnostic method in microbiology thanks to the exhaustive array of microorganisms it can detect. It appears as the next generation of tests beyond syndromic panels currently deployed in diagnostic laboratories. Nonetheless, many challenges remain to be solved before $\mathrm{CMg}$ can be widely adopted, especially the current lack of evidence that $\mathrm{CMg}$ can actually improve patient healthcare.

Acknowledgements The authors are grateful to Suhad Assad (Université Paris Est Créteil) for English revision.

\section{Declarations}

\section{Funding None.}

Conflicts of interest ER is a consultant for Pathoquest (Paris, France), and has received consultation fees from Illumina (San Diego, CA). AA received Honoraria for lectures from Gilead, for lectures and advisory board membership from Pfizer, and travel grant from Astellas. AA received expertise honoraria from Pathoquest (Paris France).

Ethics approval Not applicable.

Consent to participate Not applicable.

Consent for publication All the authors consented for publication.

Availability of data and material (data transparency) Not applicable.

Code availability (software application or custom code) Not applicable.

Authors' contributions All authors have drafted and reviewed the manuscript.

\section{References}

1. Miller S, Naccache SN, Samayoa E, Messacar K, Arevalo S, Federman S, et al. Laboratory validation of a clinical metagenomic sequencing assay for pathogen detection in cerebrospinal fluid. Genome Res. 2019;29:831-42.

2. Schlaberg R, Chiu CY, Miller S, Procop GW, Weinstock G. Validation of metagenomic next-generation sequencing tests for universal pathogen detection. Arch Pathol Lab Med [Internet]. 2017. https://doi.org/10.5858/arpa.2016-0539-RA (cited 2017 Apr 23).

3. Langelier C, Kalantar KL, Moazed F, Wilson MR, Crawford ED, Deiss $\mathrm{T}$, et al. Integrating host response and unbiased microbe detection for lower respiratory tract infection diagnosis in critically ill adults. Proc Natl Acad Sci USA. 2018;115:E12353-62.

4. Blauwkamp TA, Thair S, Rosen MJ, Blair L, Lindner MS, Vilfan ID, et al. Analytical and clinical validation of a microbial cellfree DNA sequencing test for infectious disease. Nat Microbiol. 2019;4:663-74.

5. Zhou Y, Wylie KM, El Feghaly RE, Mihindukulasuriya KA, Elward A, Haslam DB, et al. Metagenomic approach for identification of the pathogens associated with diarrhea in stool specimens. J Clin Microbiol. 2016;54:368-75.

6. Gu W, Deng X, Lee M, Sucu YD, Arevalo S, Stryke D, et al. Rapid pathogen detection by metagenomic next-generation sequencing of infected body fluids. Nat Med. 2021;27:115-24.

7. Wilson MR, Naccache SN, Samayoa E, Biagtan M, Bashir H, $\mathrm{Yu} \mathrm{G}$, et al. Actionable diagnosis of neuroleptospirosis by nextgeneration sequencing. N Engl J Med. 2014;370:2408-17.

8. Simner PJ, Miller S, Carroll KC. Understanding the promises and hurdles of metagenomic next-generation sequencing as a diagnostic tool for infectious diseases. Clin Infect Dis. 2018;66:778-88.

9. Feng L, Zhang A, Que J, Zhou H, Wang H, Guan Y, et al. The metagenomic next-generation sequencing in diagnosing central nervous system angiostrongyliasis: a case report. BMC Infect Dis. 2020;20:691.

10. Williams E, Isles NS, Seemann T, Kilpatrick T, Grigg A, Leroi $\mathrm{M}$, et al. Case report: confirmation by metagenomic sequencing of visceral leishmaniasis in an immunosuppressed returned traveler. Am J Trop Med Hyg. 2020;103:1930-3.

11. López-Labrador FX, Brown JR, Fischer N, Harvala H, Van Boheemen S, Cinek O, et al. Recommendations for the introduction of metagenomic high-throughput sequencing in clinical virology, part I: Wet lab procedure. J Clin Virol. 2021;134:104691.

12. Scharf S, Bartels A, Kondakci M, Pfeffer K, Henrich B, Haas R. Introduction of a bead beating step improves fungal DNA extraction from selected patient specimens. Int J Med Microbiol. 2020;310:151443.

13. Teng F, Darveekaran Nair SS, Zhu P, Li S, Huang S, Li X, et al. Impact of DNA extraction method and targeted 16S-rRNA hypervariable region on oral microbiota profiling. Sci Rep. 2018;8:16321.

14. Nelson MT, Pope CE, Marsh RL, Wolter DJ, Weiss EJ, Hager $\mathrm{KR}$, et al. Human and extracellular DNA depletion for metagenomic analysis of complex clinical infection samples yields optimized viable microbiome profiles. Cell Rep. 2019;26:2227-2240. e5.

15. Rodríguez A, Guillemyn B, Coucke P, Vaneechoutte M. Nucleic acids enrichment of fungal pathogens to study host-pathogen interactions. Sci Rep. 2019;9:18037.

16. Leo S, Gaïa N, Ruppé E, Emonet S, Girard M, Lazarevic V, et al. Detection of bacterial pathogens from Broncho-Alveolar lavage by next-generation sequencing. Int J Mol Sci. 2017;18(9):2011. 
17. Charalampous T, Kay GL, Richardson H, Aydin A, Baldan R, Jeanes C, et al. Nanopore metagenomics enables rapid clinical diagnosis of bacterial lower respiratory infection. Nat Biotechnol. 2019;37:783-92.

18. Angebault C, Payen M, Woerther P-L, Rodriguez C, Botterel F. Combined bacterial and fungal targeted amplicon sequencing of respiratory samples: does the DNA extraction method matter? PLoS ONE. 2020;15:e232215.

19. Graspeuntner S, Loeper N, Künzel S, Baines JF, Rupp J. Selection of validated hypervariable regions is crucial in 16S-based microbiota studies of the female genital tract. Sci Rep. 2018;8:9678.

20. Yang B, Wang Y, Qian P-Y. Sensitivity and correlation of hypervariable regions in 16S rRNA genes in phylogenetic analysis. BMC Bioinform. 2016;17:135.

21. Panek M, Čipčić Paljetak H, Barešić A, Perić M, Matijašić M, Lojkić I, et al. Methodology challenges in studying human gut microbiota-effects of collection, storage, DNA extraction and next generation sequencing technologies. Sci Rep. 2018;8:5143.

22. Heikema AP, Horst-Kreft D, Boers SA, Jansen R, Hiltemann SD, de Koning W, et al. Comparison of illumina versus nanopore $16 \mathrm{~S}$ rRNA gene sequencing of the human nasal microbiota. Genes (Basel). 2020;11(9):1105.

23. Lazarevic V, Gaïa N, Girard M, Leo S, Cherkaoui A, Renzi G, et al. When bacterial culture fails, metagenomics can help: a case of chronic hepatic brucelloma assessed by next-generation sequencing. Front Microbiol. 2018;9:1566.

24. Sanders JG, Nurk S, Salido RA, Minich J, Xu ZZ, Zhu Q, et al. Optimizing sequencing protocols for leaderboard metagenomics by combining long and short reads. Genome Biol. 2019;20:226.

25. Amos GCA, Logan A, Anwar S, Fritzsche M, Mate R, Bleazard $\mathrm{T}$, et al. Developing standards for the microbiome field. Microbiome. 2020;8:98.

26. Wilson MR, Sample HA, Zorn KC, Arevalo S, Yu G, Neuhaus $\mathrm{J}$, et al. Clinical metagenomic sequencing for diagnosis of meningitis and encephalitis. N Engl J Med. 2019;380:2327-40.

27. Sczyrba A, Hofmann P, Belmann P, Koslicki D, Janssen S, Dröge J, et al. Critical assessment of metagenome interpretation-a benchmark of metagenomics software. Nat Methods. 2017;14:1063-71.

28. Junier T, Huber M, Schmutz S, Kufner V, Zagordi O, Neuenschwander $\mathrm{S}$, et al. Viral metagenomics in the clinical realm: lessons learned from a Swiss-wide ring trial. Genes. 2019;10:655.

29. Afgan E, Baker D, Batut B, van den Beek M, Bouvier D, Cech $\mathrm{M}$, et al. The Galaxy platform for accessible, reproducible and collaborative biomedical analyses: 2018 update. Nucleic Acids Res. 2018;46:W537-44.

30. de Koning W, Miladi M, Hiltemann S, Heikema A, Hays JP, Flemming S, et al. NanoGalaxy: Nanopore long-read sequencing data analysis in Galaxy. Gigascience. 2020;9(10): giaa105.

31. Truong DT, Franzosa EA, Tickle TL, Scholz M, Weingart G, Pasolli E, et al. MetaPhlAn2 for enhanced metagenomic taxonomic profiling. Nat Methods. 2015;12:902-3.

32. Altschul SF, Gish W, Miller W, Myers EW, Lipman DJ. Basic local alignment search tool. J Mol Biol. 1990;215:403-10.

33. Langmead B, Salzberg SL. Fast gapped-read alignment with Bowtie 2. Nat Meth. 2012;9:357-9.

34. Li H. Aligning sequence reads, clone sequences and assembly contigs with BWA-MEM. arXiv:13033997 [q-bio] [Internet]. 2013 [cited 2016 Jun 15]; Available from: http://arxiv.org/abs/ 1303.3997

35. Wood DE, Salzberg SL. Kraken: ultrafast metagenomic sequence classification using exact alignments. Genome Biol. 2014; 15:R46
36. Ounit R, Wanamaker S, Close TJ, Lonardi S. CLARK: fast and accurate classification of metagenomic and genomic sequences using discriminative k-mers. BMC Genom. 2015;16:236.

37. Kim D, Song L, Breitwieser FP, Salzberg SL. Centrifuge: rapid and sensitive classification of metagenomic sequences. Genome Res. 2016;26:1721-9.

38. Nurk S, Meleshko D, Korobeynikov A, Pevzner PA. metaSPAdes: a new versatile metagenomic assembler. Genome Res. 2017:27:824-34.

39. Kolmogorov M, Bickhart DM, Behsaz B, Gurevich A, Rayko $\mathrm{M}$, Shin SB, et al. metaFlye: scalable long-read metagenome assembly using repeat graphs. Nat Methods. 2020;17:1103-10.

40. Jia B, Raphenya AR, Alcock B, Waglechner N, Guo P, Tsang KK, et al. CARD 2017: expansion and model-centric curation of the comprehensive antibiotic resistance database. Nucleic Acids Res. 2017;45:D566-73.

41. Feldgarden M, Brover V, Haft DH, Prasad AB, Slotta DJ, Tolstoy I, et al. Validating the AMRFinder tool and resistance gene database by using antimicrobial resistance genotype-phenotype correlations in a collection of isolates. Antimicrob Agents Chemother. 2019;63(11):e00483-19.

42. Bortolaia V, Kaas RS, Ruppe E, Roberts MC, Schwarz S, Cattoir V, et al. ResFinder 4.0 for predictions of phenotypes from genotypes. J Antimicrob Chemother. 2020;75:3491-500.

43. Ruppé E, Cherkaoui A, Lazarevic V, Emonet S, Schrenzel J. Establishing genotype-to-phenotype relationships in bacteria causing hospital-acquired pneumonia: a prelude to the application of clinical metagenomics. Antibiotics (Basel). 2017;6(4):30.

44. The Cryptic Consortium, The 100,000 Genomes Project. Prediction of Susceptibility to First-Line Tuberculosis Drugs by DNA Sequencing. New England Journal of Medicine. 2018;379:1403-15.

45. Bouquet J, Soloski MJ, Swei A, Cheadle C, Federman S, Billaud $\mathrm{J}-\mathrm{N}$, et al. Longitudinal transcriptome analysis reveals a sustained differential gene expression signature in patients treated for acute lyme disease. Biol. 2016;7:e0100-00116.

46. Chiu CY, Miller SA. Clinical metagenomics. Nat Rev Genet. 2019;20:341-55.

47. Naccache SN, Federman S, Veeraraghavan N, Zaharia M, Lee D, Samayoa E, et al. A cloud-compatible bioinformatics pipeline for ultrarapid pathogen identification from next-generation sequencing of clinical samples. Genome Res. 2014;24:1180-92.

48. Parize P, Muth E, Richaud C, Gratigny M, Pilmis B, Lamamy $A$, et al. Untargeted next-generation sequencing-based firstline diagnosis of infection in immunocompromised adults: a multicentre, blinded, prospective study. Clin Microbiol Infect. 2017;23:574.e1-574.e6.

49. Grumaz S, Stevens P, Grumaz C, Decker SO, Weigand MA, Hofer $\mathrm{S}$, et al. Next-generation sequencing diagnostics of bacteremia in septic patients. Genome Med. 2016;8:73.

50. Granerod J, Tam CC, Crowcroft NS, Davies NWS, Borchert M, Thomas SL. Challenge of the unknown. A systematic review of acute encephalitis in non-outbreak situations. Neurology. 2010;75:924-32.

51. Dubey D, Pittock SJ, Kelly CR, McKeon A, Lopez-Chiriboga AS, Lennon VA, et al. Autoimmune encephalitis epidemiology and a comparison to infectious encephalitis. Ann Neurol. 2018;83:166-77.

52. Thoendel MJ, Jeraldo PR, Greenwood-Quaintance KE, Yao JZ, Chia N, Hanssen AD, et al. Identification of prosthetic joint infection pathogens using a shotgun metagenomics approach. Clin Infect Dis. 2018;67:1333-8.

53. Street TL, Sanderson ND, Atkins BL, Brent AJ, Cole K, Foster $\mathrm{D}$, et al. Molecular diagnosis of orthopedic-device-related infection directly from sonication fluid by metagenomic sequencing. J Clin Microbiol. 2017;55:2334-47. 
54. Ivy MI, Thoendel MJ, Jeraldo PR, Greenwood-Quaintance KE, Hanssen AD, Abdel MP, et al. Direct detection and identification of prosthetic joint infection pathogens in synovial fluid by metagenomic shotgun sequencing. J Clin Microbiol. 2018;56(9):e00402-18.

55. Zhao M, Tang K, Liu F, Zhou W, Fan J, Yan G, et al. Metagenomic next-generation sequencing improves diagnosis of osteoarticular infections from abscess specimens: a multicenter retrospective study. Front Microbiol. 2020;11:2034.

56. Thoendel M, Jeraldo P, Greenwood-Quaintance KE, Chia N, Abdel MP, Steckelberg JM, et al. A novel prosthetic joint infection pathogen, Mycoplasma salivarium, identified by metagenomic shotgun sequencing. Clin Infect Dis. 2017;65:332-5.

57. Kollef MH. Inadequate antimicrobial treatment: an important determinant of outcome for hospitalized patients. Clin Infect Dis. 2000;31(Suppl 4):S131-138.

58. Schmidt K, Mwaigwisya S, Crossman LC, Doumith M, Munroe $\mathrm{D}$, Pires $\mathrm{C}$, et al. Identification of bacterial pathogens and antimicrobial resistance directly from clinical urines by nanopore-based metagenomic sequencing. J Antimicrob Chemother. 2017;72:104-14.

59. Pendleton KM, Erb-Downward JR, Bao Y, Branton WR, Falkowski NR, Newton DW, et al. Rapid pathogen identification in bacterial pneumonia using real-time metagenomics. Am J Respir Crit Care Med. 2017;196:1610-2.

60. Gyarmati P, Kjellander C, Aust C, Song Y, Öhrmalm L, Giske CG. Metagenomic analysis of bloodstream infections in patients with acute leukemia and therapy-induced neutropenia. Sci Rep. 2016;6:23532.

61. Horiba K, Kawada J-I, Okuno Y, Tetsuka N, Suzuki T, Ando S, et al. Comprehensive detection of pathogens in immunocompromised children with bloodstream infections by next-generation sequencing. Sci Rep. 2018;8:3784.

62. Ruppé E, Lazarevic V, Girard M, Mouton W, Ferry T, Laurent F, et al. Clinical metagenomics of bone and joint infections: a proof of concept study. Sci Rep. 2017:7:7718.

63. Rodriguez C, Jary A, Hua C, Woerther P-L, Bosc R, Desroches $\mathrm{M}$, et al. Pathogen identification by shotgun metagenomics of patients with necrotizing soft-tissue infections. Br J Dermatol. 2020;183(1):105-113.

64. Schlaberg R, Queen K, Simmon K, Tardif K, Stockmann C, Flygare $S$, et al. Viral pathogen detection by metagenomics and panviral group polymerase chain reaction in children with pneumonia lacking identifiable etiology. J Infect Dis. 2017;215:1407-15.

65. McMullan LK, Frace M, Sammons SA, Shoemaker T, Balinandi $\mathrm{S}$, Wamala JF, et al. Using next generation sequencing to identify yellow fever virus in Uganda. Virology. 2012;422:1-5.

66. Doan T, Wilson MR, Crawford ED, Chow ED, Khan LM, Knopp $\mathrm{KA}$, et al. Illuminating uveitis: metagenomic deep sequencing identifies common and rare pathogens. Genome Med. 2016;8:90.

67. Murkey JA, Chew KW, Carlson M, Shannon CL, Sirohi D, Sample HA, et al. Hepatitis E virus-associated meningoencephalitis in a lung transplant recipient diagnosed by clinical metagenomic sequencing. Open Forum Infect Dis. 2017;4:ofx121.

68. Hagiya H, Yoshida H, Shimizu M, Motooka D, Nakamura S, Iida $\mathrm{T}$, et al. Herpes zoster laryngitis in a patient treated with fingolimod. J Infect Chemother. 2016;22:830-2.

69. Frémond M-L, Pérot P, Muth E, Cros G, Dumarest M, Mahlaoui $\mathrm{N}$, et al. Next-generation sequencing for diagnosis and tailored therapy: a case report of astrovirus-associated progressive encephalitis. J Pediatric Infect Dis Soc. 2015;4:e53-57.

70. Naccache SN, Peggs KS, Mattes FM, Phadke R, Garson JA, Grant $P$, et al. Diagnosis of neuroinvasive astrovirus infection in an immunocompromised adult with encephalitis by unbiased next-generation sequencing. Clin Infect Dis. 2015;60:919-23.
71. Solomon IH, Ganesh VS, Yu G, Deng XD, Wilson MR, Miller $\mathrm{S}$, et al. Fatal case of chronic jamestown canyon virus encephalitis diagnosed by metagenomic sequencing in patient receiving rituximab. Emerg Infect Dis. 2021;27:238-42.

72. Wylie KM, Wylie TN, Buller R, Herter B, Cannella MT, Storch GA. Detection of viruses in clinical samples by use of metagenomic sequencing and targeted sequence capture. J Clin Microbiol. 2018;56(12):e01123-18

73. Fourati S, Rodriguez C, Hézode C, Soulier A, Ruiz I, Poiteau $\mathrm{L}$, et al. Frequent antiviral treatment failures in patients infected with hepatitis $\mathrm{C}$ virus genotype 4, subtype 4r. Hepatology. 2019;69:513-23.

74. Lewandowski K, Xu Y, Pullan ST, Lumley SF, Foster D, Sanderson $\mathrm{N}$, et al. Metagenomic nanopore sequencing of influenza virus direct from clinical respiratory samples. J Clin Microbiol. 2019;58(1):e00963-19.

75. Gu W, Miller S, Chiu CY. Clinical metagenomic next-generation sequencing for pathogen detection. Annu Rev Pathol. 2019;14:319-38.

76. Kufner V, Plate A, Schmutz S, Braun DL, Günthard HF, Capaul $\mathrm{R}$, et al. Two Years of Viral Metagenomics in a Tertiary Diagnostics Unit: Evaluation of the First 105 Cases. Genes (Basel). 2019;10.

77. Grad YH, Newman R, Zody M, Yang X, Murphy R, Qu J, et al. Within-host whole-genome deep sequencing and diversity analysis of human respiratory syncytial virus infection reveals dynamics of genomic diversity in the absence and presence of immune pressure. J Virol. 2014;88:7286-93.

78. Salmona M, Caporossi A, Simmonds P, Thélu M-A, Fusillier $\mathrm{K}$, Mercier-Delarue $\mathrm{S}$, et al. First next-generation sequencing full-genome characterization of a hepatitis $\mathrm{C}$ virus genotype 7 divergent subtype. Clin Microbiol Infect. 2016;22:947.e1-947. e8.

79. Fourati S, Rodriguez C, Soulier A, Donati F, Hamadat S, Poiteau L, et al. Fitness-associated substitutions following failure of direct-acting antivirals assessed by deep sequencing of fulllength hepatitis $\mathrm{C}$ virus genomes. Aliment Pharmacol Ther. 2020;52:1583-91.

80. Grubaugh ND, Ladner JT, Kraemer MUG, Dudas G, Tan AL, Gangavarapu K, et al. Genomic epidemiology reveals multiple introductions of Zika virus into the United States. Nature. 2017;546:401-5.

81. Faria NR, Quick J, Claro IM, Thézé J, de Jesus JG, Giovanetti $\mathrm{M}$, et al. Establishment and cryptic transmission of Zika virus in Brazil and the Americas. Nature. 2017;546:406-10.

82. Wu F, Zhao S, Yu B, Chen Y-M, Wang W, Song Z-G, et al. A new coronavirus associated with human respiratory disease in China. Nature. 2020;579:265-9.

83. Nilsson RH, Anslan S, Bahram M, Wurzbacher C, Baldrian P, Tedersoo L. Mycobiome diversity: high-throughput sequencing and identification of fungi. Nat Rev Microbiol. 2019;17:95-109.

84. Aykut B, Pushalkar S, Chen R, Li Q, Abengozar R, Kim JI, et al. The fungal mycobiome promotes pancreatic oncogenesis via activation of MBL. Nature. 2019;574:264-7.

85. Alanio A, Bretagne S. Pneumocystis jirovecii detection in asymptomatic patients: what does its natural history tell us? F1000Res. 2017;6:739.

86. Alanio A, Bretagne S. Challenges in microbiological diagnosis of invasive Aspergillus infections. F1000Res. 2017;6:157.

87. Irinyi L, Hu Y, Hoang MTV, Pasic L, Halliday C, Jayawardena $\mathrm{M}$, et al. Long-read sequencing based clinical metagenomics for the detection and confirmation of Pneumocystis jirovecii directly from clinical specimens: a paradigm shift in mycological diagnostics. Med Mycol. 2020;58:650-60. 
88. Zhang Y, Ai J-W, Cui P, Zhang W-H, Wu H-L, Ye M-Z. A cluster of cases of pneumocystis pneumonia identified by shotgun metagenomics approach. J Infect. 2019;78:158-69.

89. Bittinger K, Charlson ES, Loy E, Shirley DJ, Haas AR, Laughlin A, et al. Improved characterization of medically relevant fungi in the human respiratory tract using next-generation sequencing. Genome Biol. 2014;15:487.

90. Hogan CA, Yang S, Garner OB, Green DA, Gomez CA, Dien Bard J, et al. Clinical impact of metagenomic next-generation sequencing of plasma cell-free DNA for the diagnosis of infectious diseases: a multicenter retrospective cohort study. Clin Infect Dis. 2021;72(2):239-245.

91. Wilson MR, Shanbhag NM, Reid MJ, Singhal NS, Gelfand JM, Sample HA, et al. Diagnosing Balamuthia mandrillaris encephalitis with metagenomic deep sequencing. Ann Neurol. 2015;78:722-30.

92. Schneeberger PHH, Becker SL, Pothier JF, Duffy B, N'Goran $\mathrm{EK}$, Beuret $\mathrm{C}$, et al. Metagenomic diagnostics for the simultaneous detection of multiple pathogens in human stool specimens from Côte d'Ivoire: a proof-of-concept study. Infect Genet Evol. 2016;40:389-97.

93. Pfaller MA, Diekema DJ, Gibbs DL, Newell VA, Bijie H, Dzierzanowska D, et al. Results from the ARTEMIS DISK Global Antifungal Surveillance Study, 1997 to 2007: 10.5-year analysis of susceptibilities of noncandidal yeast species to fluconazole and voriconazole determined by CLSI standardized disk diffusion testing. J Clin Microbiol. 2009;47:117-23.

94. Wiederhold NP, Verweij PE. Aspergillus fumigatus and panazole resistance: who should be concerned? Curr Opin Infect Dis. 2020;33:290-7.

95. Sharma C, Nelson-Sathi S, Singh A, Radhakrishna Pillai M, Chowdhary A. Genomic perspective of triazole resistance in clinical and environmental Aspergillus fumigatus isolates without cyp51A mutations. Fungal Genet Biol. 2019;132:103265.
96. Camps SMT, Dutilh BE, Arendrup MC, Rijs AJMM, Snelders E, Huynen MA, et al. Discovery of a hapE mutation that causes azole resistance in Aspergillus fumigatus through whole genome sequencing and sexual crossing. PLoS ONE. 2012;7:e50034.

97. Spettel K, Barousch W, Makristathis A, Zeller I, Nehr M, Selitsch $\mathrm{B}$, et al. Analysis of antifungal resistance genes in Candida albicans and Candida glabrata using next generation sequencing. PLoS ONE. 2019;14:e210397.

98. Verweij PE, Ananda-Rajah M, Andes D, Arendrup MC, Brüggemann RJ, Chowdhary A, et al. International expert opinion on the management of infection caused by azole-resistant Aspergillus fumigatus. Drug Resist Updat. 2015;21-22:30-40.

99. Fraczek MG, Bromley M, Buied A, Moore CB, Rajendran R, Rautemaa R, et al. The cdr1B efflux transporter is associated with non-cyp51a-mediated itraconazole resistance in Aspergillus fumigatus. J Antimicrob Chemother. 2013;68:1486-96.

100. Sitterlé E, Coste AT, Obadia T, Maufrais C, Chauvel M, Sertour N, et al. Large-scale genome mining allows identification of neutral polymorphisms and novel resistance mutations in genes involved in Candida albicans resistance to azoles and echinocandins. J Antimicrob Chemother. 2020;75:835-48.

101. Hupalo DN, Luo Z, Melnikov A, Sutton PL, Rogov P, Escalante A, et al. Population genomics studies identify signatures of global dispersal and drug resistance in Plasmodium vivax. Nat Genet. 2016;48:953-8.

102. Doyle SR, Illingworth CJR, Laing R, Bartley DJ, Redman E, Martinelli A, et al. Population genomic and evolutionary modelling analyses reveal a single major QTL for ivermectin drug resistance in the pathogenic nematode Haemonchus contortus. BMC Genom. 2019;20:218.

103. Alanio A, Bretagne S. Difficulties with molecular diagnostic tests for mould and yeast infections: where do we stand? Clin Microbiol Infect. 2014;20:36-41. 